\title{
LA INGESTA DE ALIMENTOS DE LOS SOLDADOS CHILENOS DURANTE LA GUERRA DEL PACÍFICO (1879-1883): UNA APROXIMACIÓN HISTÓRICA Y NUTRICIONAL *
}

\author{
FOOD INTAKE OF THE CHILEAN SOLDIERS DURING THE WAR OF THE \\ PACIFIC (1879-1883): A HISTORICAL AND NUTRITIONAL APPROACH
}

\author{
Patricio Ibarra Cifuentes ${ }^{* *}$, Francisca Villavicencio Tejo ${ }^{* * *}$ y Macarena Valladares Vega ${ }^{* * *}$
}

\begin{abstract}
El objetivo de este trabajo es determinar si el tipo y la cantidad de alimentos consumidos por los soldados chilenos durante la Guerra del Pacífico (1879-1883) fueron los apropiados para cubrir sus necesidades, además de conocer parte de la rutina diaria de nutrición. La metodología utilizada fue caracterizar el tipo y calcular los ingredientes y gramos de los alimentos consumidos por los soldados, descritos en documentos oficiales chilenos, luego se calculó la cantidad de energía total diaria y macronutrientes ingeridos por los soldados. Los resultados obtenidos mostraron una mayor ingesta de energía (3.867 Kcal vs. 2.688 Kcal) y macronutrientes durante la estancia en los cuarteles que en el campamento. Hay una mayor variabilidad en el tipo de alimento durante la estancia en los cuarteles, comiendo frijoles $(350 \mathrm{~g})$, trigo picado $(120 \mathrm{~g})$ y papas frescas $(150 \mathrm{~g})$, cosas que no ingerían durante el campamento. El porcentaje de carbohidratos y proteínas consumidos por los soldados (barracas y campamentos) está dentro del rango establecido hoy, sin embargo, los niveles de lípidos están por encima de los porcentajes determinados.

Palabras claves: Guerra del Pacífico, nutrición, ingesta de energía, composición de alimentos, vida cotidiana.
\end{abstract}

The objective of this work is to determine the type and amount of food consumed by Chilean soldiers during the War of the Pacific (1879-1883), besides knowing part of the daily routine of nutrition. The methodology used was to characterize the type and calculate the ingredients and grammages of the foods consumed by the soldiers, described in chilean contemporary documents, then the amount of daily total energy and macronutrients ingested by soldiers was calculated. The results obtained showed a higher intake of energy $(3867 \mathrm{Kcal} v / \mathrm{s} 2688 \mathrm{Kcal})$ and macronutrients during the stay of the barracks than in the camp. There is a more variability in the type of food during the stay in the barracks, eating beans $(350 \mathrm{~g})$, crushed wheat (120 g) and fresh potatoes $(150 \mathrm{~g})$ things that they did not ingest during camp. The percentage of carbohydrates and proteins consumed by soldiers (barracks and camp) are within the range established today, however, lipid levels are above the determined percentages.

Key words: War of the Pacific, nutrition, energy intake, food composition, daily life.

\section{Introducción}

La movilización a gran escala producida en Chile con motivo del estallido de la Guerra del Pacífico (1879-1884), implicó que miles de hombres comunes y corrientes que se desempeñaban en las profesiones y oficios que les permitían ganarse la vida, se incorporaron al ejército que se reforzó para emprender la campaña contra el Perú y Bolivia. Se crearon nuevos regimientos y batallones de infantería, caballería y artillería, que serían los encargados de definir por la fuerza qué nación se quedaría con el control de Antofagasta y Tarapacá, donde descansaban las riquezas del salitre y el guano que fueron el motivo de la confrontación. Es así como las tropas aumentaron de 2.400 al momento del inicio de las hostilidades (febrero de 1879) (Grez 1935: 117), a cerca de 45.000 en la época de la entrada a Lima (enero de 1881) (Bulnes II 1955: 362).

Una de las consecuencias inmediatas del paso de la vida civil a la castrense para estos individuos, fue someterse a un régimen cotidiano y modos de comportamiento propios de la disciplina militar, los que fueron normados por los reglamentos y usos inherentes al oficio de las armas. Así, se vieron obligados a desarrollar determinadas habilidades de sobrevivencia y de relación con sus camaradas para intentar sortear con éxito el desafío de pervivir a la guerra (Ibarra 2015: 175). Un aspecto importante de ese cambio fue la modificación en sus hábitos

\footnotetext{
* $\quad$ Resultado Proyecto FONDECYT iniciación Nº 11160136.

** Universidad Bernardo O’Higgins, Centro de Estudios Históricos (CEH). Santiago, Chile. Correo electrónico: patricio.ibarra@ ubo.cl

*** Universidad Bernardo O’Higgins. Facultad de Salud, Departamento de Ciencias Químicas y Biológicas. Santiago, Chile. Correo electrónico: franvilla.tejo@gmail.com; macarena.valladares@ubo.cl
} 
alimenticios, en tanto se alteró la dieta a la que estaban acostumbrados, pasando a consumir lo que la milicia les entregó y lo que ellos por sus medios pudieron conseguir ${ }^{1}$.

Por su parte, el ejército chileno, en tanto institución jerarquizada y centralizada que se encargó y responsabilizó de la manutención de estos hombres mientras durara el conflicto, debió definir su política respecto de cuál sería el método más apropiado para alimentar a las tropas, además del tipo y cantidad de suministros que entregaría a sus efectivos con el objeto de establecer la ración apropiada para las necesidades del cuartel, el campamento y la marcha, lo que constituyó una problemática de mayor importancia debido a las dificultades logísticas propias de proveer alimentos durante su permanencia en territorio hostil y luego de las batallas (García 2014: 293). De la misma manera, se dio a la tarea de establecer el ordenamiento del sistema logístico adecuado para hacer llegar de manera oportuna las viandas a los distintos lugares donde fue acantonada la fuerza durante la campaña al Perú y Bolivia. Es posible señalar que esas decisiones debían tener en consideración el hecho que habría de abastecer con alimentos a un número elevado de individuos que en teoría recibirían una ración estandarizada atendiendo a las restricciones presupuestarias, la implementación logística y las características de la vida de campaña (exigencias físicas, clima, geografía, etc.) (Fatjo 2013: 1). Todo ello, en el contexto de la organización de un cuerpo armado que debió de proveerse del aparato organizativo y burocrático interno, así como de los medios y recursos necesarios para enfrentar un conflicto externo para el que no se encontraba completamente preparado, en tanto hacia comienzos de 1879 su obligación principal era cumplir con las exigencias derivadas de la mantención de la línea de frontera en La Araucanía (Grez 1935: 117). Además, el número de los soldados que efectivamente servían en la milicia iba en descenso desde ya hacía más de una década antes del estallido de las hostilidades (Verbal 2015: 115).

¿Fueron suficientes las provisiones entregadas por el ejército chileno a sus efectivos? ¿Cuál fue la cantidad de comestibles recibidos por las tropas? ¿Cumplió con las necesidades de ingesta energética de los soldados? ¿Cuándo y cómo consumieron sus comidas diarias? Estas preguntas no son contestadas a cabalidad por los escritos de Sergio Rodríguez Problemática del soldado durante la Guerra del
Pacífico (1986), el de Carlos Donoso y Juan Ricardo Couyoumdjian "De soldado orgulloso a veterano indigente. La Guerra del Pacífico" (2006) y el de William Sater Tragedia andina. La lucha en la Guerra del Pacífico 1879-1884 (2016), escritos que exploraron en las condiciones de vida y dificultades que enfrentaron los soldados durante la campaña. Tampoco en las obras de Rafael Pizarro Los abastecimientos militares en la Guerra del Pacífico (1879-1884) (1967) y de Arturo Sepúlveda Así vivieron y vencieron. 1879-1884 (1980), referidos a la logística implementada por el ejército chileno durante la Guerra del Pacífico, para hacer llegar al frente los elementos necesarios para el desarrollo de las operaciones militares. Así, a propósito de la escasa información existente en los trabajos recién nombrados, en relación con el tipo y cantidad de alimentos que consumieron los soldados chilenos durante la Guerra del Pacífico, el objetivo de este estudio es determinar las características generales de la comida que consumieron las tropas en su expedición al Perú y Bolivia. Así también, el aporte nutricional de las viandas entregadas por el ejército chileno a sus efectivos y, por medio de ello, establecer si esa ración satisfizo las necesidades de consumo de energía asociadas a la vida de campaña. Del mismo modo, se busca establecer parte de la cotidianidad y rutina de la inevitable práctica de comer. Aquello se realizará mediante una investigación interdisciplinaria que combina los métodos de la Nutrición y la Historiografía, pues para dar respuesta al problema de cómo se respondió a las necesidades cuantitativas y cualitativas de alimentación del ejército chileno en la campaña de 1879 , se requiere de una mirada instrumental y colaborativa entre lenguajes y técnicas que en apariencia son disímiles, realizando preguntas y aplicando métodos diferentes a documentos utilizados solo por la historiografía (Repko, Szostak, Buchberger 2017).

La reconstrucción del tipo y características de los alimentos que consumieron los soldados chilenos durante la Guerra del Pacífico responde a dos dimensiones que se asocian a la cotidianidad del conflicto. La primera, vinculada con las formas de satisfacer una necesidad biológica ineludible, regular y persistente: el hambre; y su materialización en el despliegue de las habilidades para cocinar y en el acto de comer en cuanto tal, los que se constituyen en prácticas culturales significativas en la construcción de lazos de asociatividad entre 
pares (Souto 2007: 129), en este caso soldados que se enfrentaron a una situación desconocida como lo fue participar de un conflicto armado que se desarrolló en territorios extraños para ellos. En segundo lugar, conocer respecto de la composición de la dieta que permitió a los soldados cumplir con las exigencias derivadas del esfuerzo físico al que estuvieron sometidos, en el entendido que debían participar obligatoriamente de las actividades propias del entrenamiento, del desplazamiento de un lugar a otro (marcha), la sobrevivencia en zonas agrestes y el combate con el adversario, entre otras.

De la misma manera, la investigación que se inicia con estas líneas permite dar indicios respecto de la alimentación de las tropas; aspecto importante de la vida cotidiana de quienes participaron del conflicto de 1879 , entendida esta como el conjunto de actividades rutinarias que los sujetos que participaron de ella realizaron a diario en diferentes esferas de acción, mediatizados por su contexto histórico y cultural (Zamora 2005: 134). Son esas prácticas habituales, en apariencia intrascendentes, las que permiten a los seres humanos conservarse como ente natural, asegurar su existencia y reproducción (Heller 1977: 19). Con todo, estas actividades se encuentran normadas por cánones de comportamiento específicos dados por su época, en este caso la circunstancia de pertenecer a una institución armada durante el desarrollo de una guerra internacional, y por ello se transforma en un hecho social en cuanto tal, del que es necesario dar cuenta y analizar (Elias 1998: 331-347). De ese modo, se intenta develar parte de las prácticas culinarias y del rito de alimentarse de los expedicionarios chilenos durante el conflicto de 1879, las que se encuentran bajo el sistema silencioso y repetitivo de servidumbres cotidianas que uno cumple por costumbre (Giard 2010: 175). Es importante destacar que esta parte de la vida del cuartel y de campaña no han sido materia de un trabajo específico que combine el método y heurística de la historiografía y la nutrición, en la búsqueda de nuevas respuestas respecto del diario vivir de las tropas.

Los antecedentes asociados a la reconstrucción y análisis de la dieta de los soldados chilenos durante la Guerra del Pacífico, fenómeno de importancia superlativa para las sociedades de los países involucrados en general y de Chile en particular, aportan al desarrollo de una historia social del conflicto, donde el estudio de la conducción político-militar y las negociaciones diplomáticas dan paso a la cotidianidad, las experiencias y problemas de las personas de carne y hueso que participaron de ella. De ese modo, se hace cargo de ilustrar un fragmento de la compleja realidad en la que se vieron involucrados los individuos que tomaron parte del conflicto de 1879 , perspectiva que no ha sido suficientemente estudiada por la historiografía (Díaz, Chaupis, Sánchez 2015: 3), pese al lugar idealizado que ocupa el roto subalterno, como uno de los gestores de la victoria en el enfrentamiento y referente identitario (Véliz 2015: 15), ocupando un lugar de privilegio en el afianzamiento de su propia autoconciencia y autovaloración positiva de la sociedad chilena (Ibarra 2015: 86). Con todo, en las últimas décadas los inquisidores del pasado han comenzado a referirse a diversas problemáticas relacionadas con la temática de guerra y sociedad (Rubilar 2015: 42).

\section{El análisis de la alimentación de las tropas chilenas en campaña: una metodología}

La información necesaria para realizar el análisis de la alimentación de los soldados chilenos de la Guerra del Pacífico fue extraída de la documentación oficial del Ejército Expedicionario que estableció detalladamente el tipo y cantidades de productos que debían ser suministrados a cada individuo ${ }^{2}$. Del mismo modo, se integró a lo anterior lo expresado en documentos personales de protagonistas de la contienda (cartas, diarios, crónicas, etc.), de los que se obtuvo datos respecto de las viandas con las que los soldados complementaron su dieta diaria y de sus formas de consumo. De estos escritos se extrajo la información necesaria para reconstruir parte de su rutina diaria, en lo referido a sus hábitos de alimentación. Se señala que a diferencia de los papeles oficiales, los testimonios permiten acceder a datos fácticos e interpretaciones respecto de lo ocurrido, desde una perspectiva única e irrepetible, pues obedece a la voluntad de su creador dar los énfasis a los hechos y reflexiones que considera dignos y necesarios de narrar para sí mismos o sus congéneres (Sarabia 1986: 191 y Castillo 2002: 25).

$\mathrm{El}$ análisis de los datos cuantitativos relativos al aporte nutritivo de los alimentos entregados a los soldados chilenos durante la Guerra del Pacífico se hizo calculando sus ingredientes y peso usando como referencia la Guía de la composición nutricional de los alimentos naturales de Vivien Gattas (2010). Se consideró el gramaje de referencia de 100 gramos 
y se calculó por regla de proporcionalidad según las cantidades señaladas por el documento que el Intendente General del Ejército y la Armada Vicente Dávila Larraín envió al ministro de la Guerra y Marina Rafael Sotomayor de enero de 1880, donde se establecieron los tipos de ración, "en marcha" y "en campamento", además de las cantidades de vituallas a entregar a cada individuo (Ahumada II 1885: 321-322). El aporte nutricional de la mayoría de los alimentos se calculó directamente desde lo establecido por el ejército considerando que eran de origen natural (papas, cebollas, ají, entre otros) y aquellos elaborados como galletas, se midió usando como referencia los ingredientes de una galleta simple hecha a base de harina (similar a la composición de un pan marraqueta).

Posteriormente se comparó la ingesta de energía y macronutrientes de las provisiones entregadas por el ejército chileno a sus soldados, basado en la referencia de alimentación normal diaria de un individuo (2.000 calorías). La operación se realizó con la distribución de la molécula calórica (DMC) propuesta por la FAO/OMS 2004 (2004) y se comparó con la alimentación de los efectivos en las dos localizaciones en las que se podían encontrar durante el desarrollo del conflicto: cuartel o campamento.

\section{El Ejército Expedicionario del Norte y la alimentación de las tropas}

El inicio de las hostilidades en febrero de 1879 sorprendió al ejército chileno sin la organización de un sistema de intendencia que permitiera una correcta distribución de los alimentos a las tropas. Tampoco contaba con la definición de cuál sería el tipo y cantidad de las raciones que recibirían los soldados que harían la expedición al Perú y Bolivia. En efecto, en términos logísticos, tanto la ocupación de Antofagasta (febrero de 1879) como la ocupación de Tarapacá (noviembre de 1879) se realizaron a base de la improvisación. Para el desarrollo de esas tareas se utilizó personal civil y se contrató proveedores particulares, los que, en algunos casos, prestaron un servicio deficiente. Aquello trajo como consecuencia la entrega a las tropas de alimentos en mal estado, con la consecuente insatisfacción de las necesidades energéticas y la aparición de distintas enfermedades entre los soldados (Bisquertt 1926: 13-14). Además, el servicio prestado fue de alto costo (Sepúlveda 1980: 22-38). Hacia mayo de 1879 se inició la organización de la Intendencia y
Comisaría General del Ejército y Armada que luego de establecida la ocupación de Tarapacá se encargaría en propiedad de la distribución de víveres, vestuario, medicinas, forraje, carbón, entre otras vituallas para las tropas en campaña (Pizarro 1967: 26). Al correr de los meses, el servicio de Intendencia fue más eficiente en su cometido. Con todo, el envío de vituallas y otros abastecimientos se articuló a partir del transporte marítimo y la implementación de bodegas de acopio en los puertos ocupados. En la medida que la campaña militar se desarrolló hacia el interior del territorio peruano, Tarapacá, Moquegua, al sur de Lima y en especial durante la incursión a la Sierra (1881-1883), la entrega de los suministros se hizo más difícil y las tropas continuaron sufriendo distintos grados de escasez de provisiones. De la misma manera, parte de esos problemas se paliaron mediante la imposición de cupos de guerra o expoliaciones a grandes, medianos y pequeños propietarios locales (Sater 2016: 95-96 y 338-362). Asimismo, esta situación de apremio para los soldados provocó que merodearan las zonas contiguas a sus lugares de acantonamiento $\mathrm{y}$, en especial, tras la batallas de mayor importancia cuando se agudizó la carestía, en busca de alimento irrumpiendo y saqueando la propiedad privada de los lugareños (Rivera 2016: 276).

En agosto de 1879, el intendente general del Ejército Francisco Echaurren escribió al general en jefe del Ejército Erasmo Escala, señalándole que en mayo de ese año solicitó un estudio para definir la ración apropiada para cada soldado. Sin embargo, en ese momento el general en jefe de la época, Justo Arteaga, no le dio importancia al tema y tampoco se pronunció al respecto. En ese mismo sentido, es factible señalar que no existen antecedentes respecto de a qué profesional o institución fue solicitado el informe (Ahumada II 1885: 43) 3 $^{3}$. Ante la indiferencia e indefinición inicial de los mandos del ejército, el tipo y cantidad de las raciones que recibieron los soldados durante la guerra, se especificó a partir de la indagación respecto de las decisiones tomadas por otros ejércitos contemporáneos. Hacia comienzos de 1880, luego de finalizada la campaña de Tarapacá y con el ejército preparándose para acometer contra la provincia peruana de Moquegua (Campaña de Tacna y Arica, marzo a junio de 1880), Vicente Dávila, intendente general del Ejército y la Armada informó al ministro de Guerra y Marina Rafael Sotomayor que los alimentos entregados a los chilenos era comparable a la recibida por los británicos durante 
la Guerra de Crimea (1853-1856), en tanto se siguió lo establecido en el escrito del general inglés Garnet Wolseley The Soldiers pocket-book for field service (1874: 53-55). Con todo, la cantidad de alimentos asignada fue modificada tras la entrada a Lima en 1881 debido al menor costo de algunos artículos. Asimismo, a propósito de las quejas de los soldados se dio más café y se agregó una pequeña dosis de aguardiente (Ahumada VI 1889: 130-131). Asimismo, eventualmente, se entregó comida enlatada (Sater 2016: 95). El tema continuó siendo de relevancia para las autoridades. En la Memoria de los trabajos ejecutados por la Intendencia Jeneral del Ejército y Armada en campaña 1880-1881, se publicó un cuadro comparativo referido a la ración que recibían los soldados en campaña de diversos ejércitos contemporáneos, información que fue extractada del Traité d'hygiene militaire de G. Morache, publicado en Paris en 1874 (Ministerio de Guerra i Marina 1882).

\section{La alimentación y su rutina: la cotidianidad de los soldados}

En el cuartel y el campamento los soldados consumieron mayoritariamente lo que el ejército les entregó. Con todo, complementaron y enriquecieron su dieta con alimentos comprados a comerciantes o recolectados en los alrededores de los lugares donde se encontraron acantonados en distintos momentos de la campaña. Fundamentalmente se trató de frutas, verduras, aves de corral, ovejas y cerdos, encontradas en las cercanías de lugares como el valle de Moquegua y Lurín. Además, lograron conseguir productos elaborados como licores o chancaca (carta de un curicano El Maipo 06/02/1881, Benavides 1967: 66, Claro 2003:70, Riquelme 1967: 46 y Urquieta I 1907: 237).

Segara los soldados las actividades diarias, tanto en el cuartel como en el campamento y la marcha, se iniciaban a las 5 de la mañana con el desayuno, el que se componía de café y pan (Del Canto 2004: 48, Olid 1999: 72 y Especial para el Diario de la Guerra Diario de la Guerra 12/06/1879). Luego comenzaba la instrucción militar, la que contempló ejercicios físicos y preparación teórica. Los primeros consistían en el perfeccionamiento de los movimientos en grupo, las técnicas de la lucha en guerrilla, el uso de la bayoneta y el tiro al blanco, entre otros. Los segundos, en el estudio de la ordenanza militar, tácticas y el uso de las distintas armas. Tras el entrenamiento, las tropas almorzaban un plato elaborado generalmente a partir de charqui, porotos, trigo, cebollas, papas, grasa y ají, en cualquiera de las combinaciones posibles (Mason 1971: 59-60). Según la opinión del corresponsal de La Patria, en mayo de 1879 escribiendo desde Antofagasta, $L a$ alimentación del soldado es buena. Bien condimentada y hecha con buenos artículos (Castagneto 2015: 174). Tras el receso de mediodía había un recreo, para luego volver a los ejercicios hasta eso de las 6 de la tarde (Del Canto 2004: 48). La última comida del día consistía en un "plato de carne y un pan" (Especial para el Diario de la Guerra Diario de la Guerra 12/06/1879). Más tarde, quedaban en libertad hasta las 9 de la noche cuando se tocaba la retreta. En ese intervalo de tiempo recorrían la ciudad donde se encontraban, y en ocasiones, dependiendo del dinero que poseían, consumían alcoholes como chicha, cerveza, destilados o vino, como ocurrió mientras duró el acantonamiento en Antofagasta (Venegas 1885: 23). Mediante el relato del soldado del batallón Chillán Hipólito Gutiérrez, se sabe que también hubo personal especial, denominados "Rancheros", que cocinaron para las tropas. En su relato de su experiencia en la guerra, el propio Gutiérrez aseguró que mientras se encontró con sus camaradas en Antofagasta "lo pasamos bien, bien comidos, y buen café que los daban por la mañana, y tres panes bien regulares que los daban al día y entonces y buena comida de carne y legumbres" (Gutiérrez 1976: 166).

En campaña, los hombres luego de la instrucción militar se dedicaban a diversas tareas domésticas, así como el aseo de sus habitaciones o tiendas, el lavado de ropa, entre otras. Recibían una "ración de campamento", la que se componía de porotos, charqui, harina tostada, galleta, grasa, cebollas, ají y sal. Con el correr del tiempo y aprovechando la experiencia adquirida se creó la "Ración fresca de campamento" consistente en carne, papas, frangollo (maíz triturado para cocerlo) o arroz y café, además de cebollas, ají, sal, galleta, harina tostada y cebollas que ya eran parte de la dieta de los soldados ${ }^{4}$. Los animales destinados a dar carne fresca llegaban desde Chile y eran sacrificados in situ, a razón de 50 bueyes por semana. Su distribución solo se realizaba luego de asentada la ocupación de los territorios conquistados (Ahumada II 1885: 321) . En caso de carestía de vacunos, hubo ocasiones en que se echó mano a la carne de burro como sucedió en el vivac de Las Yaras, mayo de 1880, durante la 
campaña de Tacna y Arica (Dublé 1968: 128-129, Castro 1986: 24). Asimismo, "doscientos cincuenta gramos de carne fresca podían ser sustituidos por charqui y porotos" (Mason 1971: 62). Los alimentos eran cocinados por ellos mismos. Normalmente se agrupaban en "carretas" para compartir y hacer rendir mejor las viandas que poseían, por cuanto la escasez de productos era la norma. En efecto, el soldado Gutiérrez anotó en sus memorias que en Las Yaras (Moquegua, Campaña de Tacna y Arica mayo de 1880) "Harto tuvimos que sufrir de comidas", debido a los problemas en la distribución de los suministros entregados por el ejército (Gutiérrez 1976: 186). Por su parte, el subteniente Arturo Benavides rememoró que previo a la batalla del Campo de Alianza (26 de mayo de 1880) le obsequiaron en varias "carretas" "sopaipillas pasadas, café con leche condensada, que era un lujo, y variados comestibles" (Benavides 1967: 66). Es posible señalar que los tipos de platos y alimentos cocinados por la tropa tenían su origen en las formas de la cocina criolla chilena heredada de la tradición colonial (Pereira 1977) y de las influencias extranjeras recibidas durante la vida republicana (Páez 1998: 366).

Por su parte, a lo largo de la guerra el licor fue escaso repartiéndose entre pocos (Medina 1952:13 y Cartas de la campaña [correspondencia especial de La Patria], La Patria 16/02/1880). Sin embargo, los oficiales asumieron la necesidad de contar siempre con vino y aguardiente, debido a los beneficios que podían traer en la cotidianidad del campamento. Según la Cartilla del soldado de 1879 , en caso de ser imprescindible beber "aguas malas", es decir turbias, debían "mezclarse con vino aguardiente o vinagre". Del mismo modo, en invierno "el aguardiente tomado en pequeñas cantidades es una excelente bebida en las noches frías y húmedas" y "en verano es necesario agregarle 6 o 7 partes de agua" (1879: 52).

También existió una ración para el soldado "en marcha", es decir, para cuando se desplazaban a pie por largas distancias para llegar a los lugares donde acamparían, o bien, buscarían al adversario y para combatirlo. Esta consistió en charqui, harina tostada, galleta, ají y cebolla (Ahumada II 1885: 321) ${ }^{6}$.

\section{Cuantificación de la ingesta energética de los soldados}

Como se mencionó, durante el desarrollo de la Guerra del Pacífico los soldados mayoritariamente permanecieron en un cuartel (ciudad) o en el campamento (campaña). A continuación se describirá la ingesta energética en ambas situaciones.

\section{Ingesta energética durante la permanencia en el cuartel}

En esta etapa los soldados consumieron principalmente alimentos ricos en carbohidratos como trigo, arroz, harina y papas. También se observa un alto consumo de legumbres como los porotos (Tabla 1). Adicionalmente, se observa que la principal fuente de proteínas, carbohidratos y lípidos provenían del charqui, harina y manteca, respectivamente. Se observa que durante la permanencia en el cuartel el principal consumo de energía provenía de la harina (696 Kcal), luego de alimentos como las galletas, charqui y porotos. De esta manera, durante dichas jornadas, el consumo total de energía de los soldados correspondía a 3.867,5 Kcal. Adicionalmente, se observa un bajo consumo de alimentos frescos que correspondían a $314 \mathrm{Kcal}$ diarios. De los documentos revisados para la presente investigación se observa que los oficiales recibían una ración de arroz adicional. Por esa razón, en la Tabla 1 los valores se encuentran calculados en ambos casos.

\section{Ingesta energética durante la permanencia en el campamento}

Durante la estancia en el campamento se muestra que el consumo energético total por soldado era de $2.688,7 \mathrm{Kcal}$ (Tabla 2), donde el mayor aporte proviene del consumo de carbohidratos, contenidos en alimentos como galletas y seguido del trigo tostado. Asimismo, la principal fuente de proteínas y de lípidos correspondía a charqui.

Por otro lado, el estudio de la cantidad de energía y macronutrientes aportados por los alimentos frescos muestra que el mayor aporte calórico y de macronutrientes provino de los frijoles. Además, se observa el consumo de tres tipos de verduras (papa, cebolla y zanahoria) y el resto de los alimentos frescos corresponde a legumbres, cereales y carne.

\section{Comparación entre la ingesta nutricional de los soldados y lo estipulado por la FAO/OMS}

Al comparar la ingesta energética de los soldados de acuerdo con lo estipulado por la FAO/OMS 
Tabla 1. Composición de alimentación de los soldados durante la Guerra del Pacífico en el cuartel.

\begin{tabular}{|c|c|c|c|c|c|}
\hline Tipo de alimento & $\begin{array}{c}\text { Cantidad } \\
\text { entregada }(\mathrm{g})\end{array}$ & Energía (Kcal) & Proteínas (g) & Carbohidratos (g) & Lípidos (g) \\
\hline Charqui & 150 & 531 & 94,8 & 9,15 & 12,75 \\
\hline Porotos & 350 & 490 & 33,95 & 82,6 & 2,45 \\
\hline Trigo machacado (mote) & 120 & 166,8 & 4,2 & 36,4 & 0,48 \\
\hline Arroz (oficiales) cocido & 120 & 155 & 3,2 & 33,4 & 0,36 \\
\hline Galleta & 200 & 544 & 12,8 & 120 & 1,4 \\
\hline Harina & 200 & 696 & 8 & 152,8 & 2,4 \\
\hline Trigo tostado & 200 & 414 & 11,1 & 86,4 & 2,4 \\
\hline Cebollas & 50 & 21,5 & 0,4 & 4,8 & 0,1 \\
\hline Manteca & 50 & 445,5 & 5 & 0 & 49,5 \\
\hline Ají & 5 & 2 & 0,1 & 0,3 & 0 \\
\hline Sal & 15 & & & & \\
\hline Papas & 150 & 117 & 3,9 & 25,05 & 0,15 \\
\hline Azúcar & 25 & 100 & 24,8 & 0 & 0 \\
\hline Café & 10 & 26,1 & 1,6 & 4,9 & 0,01 \\
\hline total & & 3708,9 & 203,85 & 555,8 & 72 \\
\hline $\begin{array}{l}\text { Total menos el arroz } \\
\text { (oficiales) }\end{array}$ & -120 & 3553 & 200,65 & 522,4 & 71,6 \\
\hline *Más la comida fresca & +230 & 314,5 & 19,06 & 52,5 & 1,77 \\
\hline Total & & 3867,5 & 219,06 & 574,9 & 73,37 \\
\hline
\end{tabular}

Tabla 2. Composición nutricional de los soldados en el campamento.

\begin{tabular}{lccccc}
\hline \multicolumn{1}{c}{ Tipo de alimentos $(\mathrm{g})$} & & Energía $(\mathrm{Kcal})$ & Proteínas $(\mathrm{g})$ & Carbohidratos $(\mathrm{g})$ & Lípidos $(\mathrm{g})$ \\
\hline Charqui & 460 & 531 & 94,8 & 9,15 & 12,75 \\
Galleta & 460 & 1251,2 & 29,44 & 276 & 3,22 \\
Trigo tostado & 200 & 414 & 11,1 & 86,4 & 2,4 \\
Cebollas & 100 & 43 & 0,8 & 9,6 & 0,2 \\
Ají & 10 & 4 & 0,2 & 0,6 & 0 \\
Manteca & 50 & 445,5 & 5 & 0 & 49,5 \\
Total & & 2688,7 & 141,34 & 381,75 & 68,07 \\
\hline
\end{tabular}

Tabla 3. Distribución de la molécula calórica indicaciones de la FAO / OMS y soldados durante la Guerra del Pacífico.

\begin{tabular}{lccc}
\hline \multicolumn{1}{c}{ Macronutriente $(\%)$} & FAO / OMS 2004 & Soldados en cantón & Soldados en la marcha \\
\hline Carbohidratos & $50-60$ & 59 & 56,5 \\
Lípidos & $12-16$ & 23 & 20,9 \\
Proteínas & $25-30$ & 17 & 22 \\
\hline
\end{tabular}

(2004) se observa que el porcentaje de carbohidratos y proteínas están dentro del rango establecido. Sin embargo, los niveles de lípidos están por sobre los porcentajes determinados, dichas comparaciones se observan en la Tabla 3.

\section{Discusión}

El presente escrito muestra una descripción y análisis cualitativo y cuantitativo respecto de la alimentación de las tropas durante la Guerra del Pacífico. Este tipo de estudio solo es posible de realizar a partir de la información contenida en la documentación donde se establece el tipo y cantidad de viandas a entregar a cada soldado (Ahumada II 1885: 321 y VI 1889: 130-131); para luego proceder a la cuantificación de sus macro y micronutrientes.

Del análisis realizado llama la atención que durante la permanencia de los soldados en el cuartel existía un mayor consumo de calorías y 
de macronutrientes que durante la estancia en el campamento, considerando que las actividades eran más demandantes en esta última situación. De lo anterior podría inferirse que se debió a la dificultad para trasladar variedad y cantidad de alimentos, además de mantenerlos en condiciones óptimas durante la estancia en el campamento. Por otra parte, la composición de la ración de campamento permitía ahorrar espacio y peso, tanto para los soldados como para quienes las transportaban al vivac. Adicionalmente, se observa en ambas situaciones poca variedad en las provisiones entregadas. La mayoría corresponde a alimentos no perecibles como azúcar, café, sal, harina, legumbres y charqui, además de otros que se pueden conservar por más tiempo a temperatura ambiente (Wolseley 1874: 53-55 y Ahumada VI 1889: 130-131). Aquello responde a la necesidad de transportar grandes cantidades de pertrechos desde el Chile central a Antofagasta, Iquique, Arica, Tacna o Lima debiendo cubrir largas distancias por mar y tierra. Por otro lado, durante la estancia en el cuartel los soldados tuvieron acceso a alimentos frescos, entre ellos zanahorias, los que se consumieron irregularmente en el campamento.

Como ya se señaló, de acuerdo con documentación consultada las autoridades chilenas encargadas de la conducción del conflicto de 1879 se hicieron cargo de satisfacer las necesidades alimenticias de las tropas equiparando las raciones que entregaron con lo establecido en el Soldier's pocket-book for field service, escrito por el mayor general inglés Wolseley en 1874 (Ahumada II 1885: 321 y VI 1889: 130-131). Es admisible señalar que se entiende por requerimientos nutricionales como la ingesta (a partir de la dieta: alimentos y bebidas, incluida el agua) que, teniendo en cuenta la información disponible acerca de la distribución de los requerimientos en un grupo de personas, se juzga apropiada para mantener la salud de prácticamente todos los individuos sanos del grupo (FAO 2004). Evidentemente, hacia el último cuarto del siglo XIX la bromatología, denominación que en esa época se le dio a la disciplina dedicada al estudio de los alimentos, sus componentes y propiedades, no había establecido dichos requerimientos, como sí lo están hoy. En definitiva, a la luz de estos antecedentes la decisión tomada por las autoridades chilenas de diseñar una ración estandarizada basándose en la experiencia de otros ejércitos contemporáneos fue la más adecuada.

Resulta interesante señalar que durante la estancia en el cuartel el mayor aporte calórico provenía de la harina y del charqui. En cambio, durante la estancia en el campamento fueron el charqui y los porotos quienes aportaron gran cantidad de energía. En ese sentido, ambos alimentos entregan un alto aporte proteico, lo que apoya al desarrollo de masa muscular (Gorrissen, Rémond y van Loon 2015: 96100), aspecto importante para el desarrollo de las actividades cotidianas de los soldados, considerando el rigor del clima, las dificultades de los caminos y la posibilidad de combatir al enemigo durante el desplazamiento de un punto a otro.

Por otro lado, se observa que las raciones de alimentos entregadas a cada soldado cumplen con los actuales requerimientos establecidos por la FAO/OMS (2004), a excepción de los lípidos. Sin embargo, dichas recomendaciones son estipuladas para poblaciones o grupo de personas sin considerar actividades físicas de alta demanda, que impliquen un mayor gasto energético, como las realizadas durante una guerra. Es importante destacar que debido a la alta carga de esfuerzo físico a la que estaban sometidas las tropas, dichos requerimientos nutricionales debían ser suficientes para cumplirlas. Actualmente existen evidencias que muestran que las raciones de combate administradas a los hombres por más de 34 días producirían disminución del peso y composición corporal donde disminuiría la masa grasa y muscular (Tassone 2017: 897-910).

Los documentos analizados no se refieren al estado sanitario de los soldados, en temáticas como el peso y otros. Por tanto, no fue posible asociar la descripción y cuantificación de la alimentación con aspectos de salud. La información contenida en esos escritos permitió cuantificar en energía total y por macronutrientes que tuvieron las tropas chilenas durante la Guerra del Pacífico. Actualmente, los instrumentos más utilizados para determinar ingesta diaria son: registro de alimentos, registro gráfico, por pesada, tendencia de consumo, recordatorio de 24 horas, entre otras. Sin embargo, todas tienen algunas dificultades, ya que cuantificar la ingesta diaria individual supone la consideración de diversas variables y problemas (FAO 2004).

\section{Conclusión}

Superada la indefinición que caracterizó la organización del ejército chileno al momento del estallido de la Guerra del Pacífico, el servicio de Intendencia General del Ejército y Marina se 
dio a la tarea de estipular lo que fue la ración de alimentos estandarizada para los soldados que hicieron la campaña a Bolivia y Perú entre 1879 y 1883. De esta manera, las autoridades a cargo de la conducción del ejército expedicionario al Perú y Bolivia comprendieron la necesidad de entregar a sus hombres la mejor ración de alimentos posible con los elementos con que se contaba entre 1879 y 1884, por cuanto un soldado al que se le entregan los requerimientos nutricionales óptimos, podrá desempeñarse de mejor manera frente a las exigencias que las circunstancias asociadas a la campaña militar les impusiera. Con el objeto de tomar la mejor decisión posible, la jefatura se basó en la experiencia de los ejércitos europeos de la época, en especial el británico, y a partir de allí estableció el patrón nutritivo que serviría de referencia durante el desarrollo de las operaciones del Ejército Expedicionario.

La decisión tomada por las autoridades de la guerra, en cuanto a la ración que debía recibir cada soldado, fue apropiada, por cuanto ella cumplió con los requerimientos energéticos necesarios para la vida de cuartel y de campaña. Sin embargo, se trata de una porción teórica y no necesariamente fue lo que recibieron las tropas, por cuanto en la bibliografía relativa a la vida de campaña, a la implementación del sistema logístico del ejército y en los documentos personales de los combatientes, existen referencias que indican que los suministros no siempre llegaron en la cantidad, condiciones y oportunidad requerida.

Los resultados obtenidos en este estudio, cuantitativos y cualitativos, permiten recabar antecedentes respecto de cómo se resolvió por parte de los directores chilenos de la guerra, la problemática asociada a la satisfacción de las necesidades alimenticias en virtud de la exigencias del esfuerzo físico en la cotidianidad de las tropas (cuartel y campamento) y los requerimientos específicos para la marcha entre lugares alejados y a campo traviesa. Además, a partir del uso de documentos personales de protagonistas de la Guerra del Pacífico permitió establecer parte de la rutina diaria de nutrición y en qué condiciones se consumieron los alimentos proporcionados a los soldados.

En definitiva, este escrito es una contribución a analizar y entender aspectos relacionados con la historia social y la vida cotidiana de las tropas chilenas durante los años de la Guerra del Pacífico, desde una perspectiva multidisciplinaria integrando las herramientas metodológicas de la Historia y la Nutrición. De ese modo, permite ampliar la mirada para de ese modo explorar en nuevas temáticas e interpretaciones respecto de un hito de gran trascendencia para el Chile contemporáneo.

\section{Referencias Citadas}

1879 Cartilla del soldado. Imprenta Sociedad Instrucción Primaria, Santiago.

1880 Memoria para optar al grado de licenciado en medicina de Ernesto Turenne: Higiene naval, aplicada a la Guerra del Pacífico en Anales de la Universidad de Chile, 57, 1: 325-340.

Ahumada, $\mathrm{P}$.

1884-1892 Guerra del Pacífico. Recopilación completa de todos los documentos oficiales, correspondencias y demás publicaciones que ha dado a la luz la prensa de Chile, Perú y Bolivia, conteniendo documentos inéditos de importancia 8 Volúmenes, Imprenta i Lib. Americana de Federico T. Lathrop, Valparaíso.

Benavides, A.

1967 Seis años de vacaciones. Editorial Francisco de Aguirre, Buenos Aires.

Bisquertt, $\mathrm{M}$.

1926 "Resumen histórico del desarrollo de los servicios de alimentación en campaña”. Revista del servicio administrativo del Ejército chileno 1: 7-16.

Bulnes, G.

1955 Guerra del Pacífico. 3 Volúmenes, Editorial del Pacífico, Santiago.
Carta de un curicano, El Maipo 06/02/1881, San Bernardo. Cartas de la campaña. (Correspondencia especial de La Patria). La Patria 16/02/1880, Valparaíso.

Castillo, A.

2002 "De la suscripción a la necesidad de escribir". En $\mathrm{La}$ conquista del alfabeto. Escritura y clases populares, A. Castillo. (Coordinador) pp. 21-51. Ediciones Trea, Gijón. Castagneto. P.

2015 Corresponsales en campaña en la Guerra del Pacífico. 1879-1881. RIL Editores, Santiago.

Castro, G.

1986 Guerra del Pacífico. Diario de campaña. 1880-1881. Santiago.

Claro, $\mathrm{R}$.

2003 "Cartas de don Máximo R. Lira a doña Isabel Errázuriz desde los campamentos durante la Guerra del Pacífico (1879-1881)". En Historia 36: 61-88.

Del Canto, E.

2004 Memorias militares. Centro de Estudios Bicentenario, Santiago.

Díaz, A; Chaupis, J.; Sánchez, E.

2015 "La otra Guerra del Pacífico". En Diálogo Andino 48: $3-5$. 
Donoso, C. y J. Couyoumdjian

2006 "De soldado orgulloso a veterano indigente. La Guerra del Pacífico". En Historia de la vida privada en Chile, Vol. 2, R. Sagredo y C. Gazmuri (Directores), pp. 237-273. Taurus, Santiago.

Dublé Almeyda, D.

1968 "La jornada de Tacna". En Revista Chilena de Historia y Geografía, 136: 125-158.

Elias, N.

1998 "Apuntes sobre el concepto de lo cotidiano". En $\mathrm{La}$ civilización de los padres y otros ensayos. Weiler, V. (Comp. \& Trad.), Grupo Editroial Norma, Bogotá.

Especial para el Diario de la Guerra, Diario de la Guerra 12/06/1879, Santiago.

Expedición chilena sobre Moquegua. Octubre de 1880. Diario llevado por orden del jefe de la expedición Sr. Juan de la Cruz Salvo, Archivo Nacional Histórico, Fondo Benjamín Vicuña Mackenna, Vol. 246: Fojas 1-70.

FAO. Recomendaciones de DMC (FAO-OMS 2004).

Fatjo, P.

2013 Introducción a la alimentación militar en España (18591914). Universitat Autónoma de Barcelona, Barcelona.

García, A.

2014 "La alimentación del Ejército de Tierra en operaciones. La ración individual de combate". En Sanidad Militar, 70 (4): 293-306.

Gattás, V.

2010 Guía de la composición nutricional de los alimentos naturales, de la industria y preparaciones chilenas habituales, Universidad de Chile-INTA, Santiago.

Giard, L.

2010 "Hacer de comer". En La invención de lo cotidiano 2. Habitar, cocinar. De Certaeau, M, Giard, L. y Mayol, P. Universidad Iberoamericana, Instituto Tecnológico y de Estudios superiores de occidente, México D. F.: 151-255.

Gorissen, SH; Rémond, D. y van Loon, LJ.

2015 "The muscle protein synthetic response to food ingestion". Meat Sci 109: 96-100.

Grez, C.

1935 "La supuesta preparación de Chile para la Guerra del Pacífico". Boletín de la Academia Chilena de la Historia 5: 111-139.

Gutiérrez, H.

1976 "Crónica de un soldado de la Guerra del Pacífico". En Dos soldados en la Guerra del Pacífico. Gutiérrez, H. y Quiroz, A. Editorial Francisco de Aguirre S. A., Buenos Aires: 157-229.

Heller, A.

1987 Sociología de la vida cotidiana, Ediciones Península, Barcelona.

Ibarra, P.

2015 “"El Perú y Bolivia ante el General Pililo"”: los enemigos de Chile en las caricaturas de la Guerra del Pacífico (1879-1883)". Diálogo Andino 48: 85-95.

Ibarra, $P$.

2015 "En Pacocha y Lima: dos epístolas de Alberto del Solar durante la Guerra del Pacífico (1880-1881)". Revista de Historia y Geografía 33. 175-188.

Le León, M.

1969 Recuerdos de una Misión en el Ejército Chileno. Batallas de Chorrillos y Miraflores. Con resumen de la Guerra del
Pacífico y notas, Editorial Francisco de Aguirre, Buenos Aires.

Medina, J.

1952 Una excursión a Tarapacá. Los Juzgados de Tarapacá. 1880-1881. Dirección General de Prisiones, Santiago.

Mason, T.

1971 Guerra del Pacífico. Editorial Francisco de Aguirre, Buenos Aires-Santiago.

Ministerio de Guerra i Marina

1882 Memoria de los trabajos ejecutados por la Intendencia Jeneral del Ejército y Armada en Campaña. 1880-1881. Imprenta de la Época, Santiago.

Morache, G.

1874 Traité d'hygiene militaire. Libraire J.-B. Bailliére et fils. Paris.

Olid, A.

Crónicas de guerra. Relatos de un ex combatiente de la Guerra del Pacífico y la Revolución de 1891. RIL Editores, Santiago.

Páez, R.

"Alimentos, vida cotidiana y modernidad en sociedad provinciales del siglo XIX: El afrancesamiento gastronómico en La Serena y Concepción", Chile En Anuario IEHS 13: 357-367.

Palma, D.

"De apetitos y de cañas. El consumo de alimentos y bebidas en Santiago a fines del siglo XIX". En Historia 37, II: 391-417.

Pereira, E.

1977 Apuntes para la historia de la cocina chilena. Editorial Universitaria, Santiago.

Pizarro, R.

1967 Los abastecimientos militares en la Guerra del Pacífico (1879-1884). Ministerio de Defensa Nacional, Biblioteca del Oficial, Santiago.

Ramírez, A.

1850 "Minería Traducción de la memoria del señor Gasparín presentada a la Academia de Paris, sobre el régimen de los mineros belgas". En Anales de la Universidad de Chile: 333-338.

Repko, A; Szostak, R; Philips, M.

2017 Introduction to interdisciplinary studies. SAGE Publications. Los Angeles.

Riquelme, D.

La expedición a Lima. Editorial del Pacífico, Santiago.

Rivera, $\mathrm{P}$.

2016 "Fantasmas de rojo y azul. Los saqueos de las tropas chilenas en la Guerra del Pacífico". En Anuario Colombiano de Historia Social y de la Cultura, 43, 1: 263-293.

Rodríguez, $\mathrm{S}$.

1986 Problemática del soldado durante la Guerra del Pacífico. Edimpres Ltda., Santiago.

Rubilar, M.

2015 "Prensa e imaginario nacional: la misión social de los actores subalternos regionales durante la Guerra del Pacífico”. En Diálogo Andino 48: 41-53.

Sater, W.

2016 Tragedia andina. La lucha en la Guerra del Pacífico. 1879-1884. Centro de Investigaciones Diego Barros Arana, Santiago.

Sarabia, B.

1986 "Documentos personales: historias de vida". En El análisis de la realidad social. Métodos y técnicas de 
investigación social, García, M; Ibáñez, J., \& Alvira, F. (Editores), pp. 187-208, Alianza Editorial, Madrid.

Sepúlveda, A.

1980 Así vivieron y vencieron. 1879-1884. Impresos Esparza y Cía. Ltda., Santiago.

Souto, M.

"Sobre los festines y el hambre en Nueva España". En Gozos y sufrimientos en la historia de México, P. Gonzalbo y V. Zárate (coordinadores), El Colegio de México, Instituto Mora, México D. F.

Tassone, E. y Baker, B.

2017 Body weight and body composition changes during military training and deployment involving the use of combat rations: a systematic literature review. Br J Nutr 117 (6): 897-910.

Urquieta, A.

1908-1909 Recuerdos de la vida de campaña en la Guerra del Pacífico. II Tomos. Imprenta, Calle Juarez, Santiago.
Véliz, C. Bajo la tienda

1958 "La representación subalterna del 'roto' como fundamento de nacionalidad para el siglo XX chileno". En Diálogo Andino 48: 7-17.

Venegas. L.

1885 Sancho en la guerra. Recuerdos del ejército en la campaña del Perú y Bolivia. Imprenta Victoria, Santiago.

Verbal, V.

2015 "El ejército de Chile en vísperas de la Guerra del Pacífico. Los problemas del enganche (1866-1879)"'. Revista de Historia 22: 111-136.

Wolseley, G.

1874 The Soldiers pocket-book for field service. Macmillan and Co., London, England.

$\mathrm{Wu}, \mathrm{C}$.

1986 Testimonios británicos de la ocupación chilena de Lima (enero de 1881), Editorial Milla Batres, Lima.

Zamora, I.

2005 "La importancia de la vida cotidiana en los estudios antropológicos". Revista Líder 14, 10: 123-143.

\section{Notas}

1 La alimentación cotidiana de los individuos pertenecientes al bajo pueblo durante su vida civil, quienes en su mayoría conformaron el grueso de las tropas expedicionarias al Perú y Bolivia, consistió en platos tradicionales y económicos como los porotos, el charquicán, pequenes, pan de grasa, chancho arrollado, ajiaco, cazuela de ave, carbonada, empanadas, chanfaina y humitas, entre otros, además de frutas y verduras de la estación (Palma 2004: 399).

2 "Nota del Intendente general del ejército i armada al Ministro de la Guerra, relativa al abastecimiento del ejército en campaña" (Ahumada II 1885: 321-322), Memoria del delegado de la Intendencia Jeneral del Ejército i Armada, don Hermójenes Pérez de Arce (Ahumada VI 1889: 130131) y Documento $\mathrm{N}^{\circ} 13$ (Ministerio de Guerra i Marina 1882)

3 Durante el siglo XIX la bromatología, disciplina dedicada al estudio de las propiedades y características de los alimentos, tuvo cierta cabida en la discusión científica del Chile de la segunda mitad del siglo XIX. En los Anales de la Universidad de Chile se publicaron algunos trabajos que contenían referencias a la materia. Véase Antonio Ramírez: Minería. Traducción de la memoria del señor Gasparín presentada a la Academia de Paris, sobre el régimen de los mineros belgas (1850: 3339-338), y la Memoria para optar al grado de licenciado en medicina de Ernesto Turenne: Higiene naval, aplicada a la Guerra del Pacífico (57, 1, 1880: 325-340).

4 Según el testimonio de los observadores contemporáneos extranjeros que acompañaron al ejército chileno durante la campaña, Wiiliam Dyke Acland (Inglés), M. León (Francés) y Theodorus B. M. Mason (Estadounidense) en condiciones normales en el campamento y la marcha, la cantidad de alimentos entregados a las tropas era muy similar a la señalada en los documentos citados (Wu 1986:62, Le León 1969: 211, Mason 1971: 60-61).

5 Según el testimonio del médico Guillermo Castro, los heridos y enfermos recibían prácticamente la misma cantidad de alimento que los soldados sanos y en varias oportunidades también escasearon las viandas para ellos. Castro anotó en su Diario de campaña el 19 de mayo de 1880 que: No se puede conseguir un gramo de arroz para los enfermos. Dos días después aseguró que La ambulancia de la división no tiene chuño ni linaza. Por otra parte, el 23 de diciembre de 1880 señaló que recibieron víveres para 10 días - para 50 enfermos 2 líos de charqui - 1 saco de arroz, cuatro sacos harina, 1 café - 2 azúcar rubia - sal grasa 5 latas (Castro 1986: 24 y 59-60).

6 Por ejemplo, José M. Walker escribió a Benjamín Vicuña Mackenna que la columna chilena que combatió en el puente Topater el 23 de marzo de 1879, primera acción terrestre de la guerra, salió perfectamente dotada, pues Después de una buena comida de carne los soldados cargaron en sus mochilas víveres secos para dos días de marcha (Castagneto 2015: 93). Asimismo, la expedición a Moquegua encabezada por Juan de la Cruz Salvo en octubre de 1880 llevó como víveres 29 animales vacunos, 20 quintales de harina, 15 quintales de harina tostada, 10 sacos de cebada y 98 barriles para agua (expedición chilena sobre Moquegua. Octubre de 1880. "Diario llevado por orden del jefe de la expedición Sr. Juan de la Cruz Salvo", Archivo Nacional Histórico, Fondo Benjamín Vicuña Mackenna, Vol. 246, Fojas 12 y 12 vuelta). 
